\title{
Reprojeto de um dispositivo eletromecânico em uma abordagem de engenharia reversa integrada ao projeto para manufatura e montagem e à prototipagem rápida
}

\author{
Carlos Henrique Pereira Mello ${ }^{\mathrm{a} *}$, Fabrício Oliveira de Toledo ${ }^{\mathrm{b}}$, Dóris Akemi Akagic, \\ José Hamilton Chaves Gorgulho Júnior ${ }^{\mathrm{d}}$, Amanda Fernandes Xavier \\ a*carlos.mello@unifei.edu.br, UNIFEl, Brasil \\ bfabricio696@yahoo.com.br, UNIFEl, Brasil \\ cakemiakagi@yahoo.com.br, UNIFEl, Brasil \\ dgorgulho@unifei.edu.br, UNIFEl, Brasil \\ eamandaxavier@unifei.edu.br, UNIFEl, Brasil
}

\begin{abstract}
Resumo
0 tema deste trabalho é o estudo da integração do projeto para manufatura e montagem com a prototipagem rápida, em uma abordagem de engenharia reversa, como ferramenta de suporte ao reprojeto de produtos. A partir de uma fundamentação teórica sobre esses conceitos, o presente trabalho visa analisar a aplicação de um modelo para a utilização integrada do projeto para manufatura com a prototipagem rápida em uma abordagem da engenharia reversa no processo de reprojeto de produtos eletromecânicos e analisar os resultados da aplicação do referido modelo para a redução do tempo de produção/montagem e dos custos de manufatura/montagem no reprojeto desse produto que está, há mais de 30 anos, no mercado. 0 método de pesquisa empregado foi a pesquisa-ação, uma vez que o pesquisador buscava resolver um problema identificado dentro do objeto de estudo em parceria com a equipe de profissionais da empresa. Os resultados das recomendações para o reprojeto do produto apresentam uma redução no tempo de montagem, do número de processos e de componentes e no custo-meta do produto, propiciando a oportunidade de melhorar a sua competitividade no mercado.
\end{abstract}

Palavras-chave

Engenharia reversa. DFMA. Prototipagem rápida. Reprojeto.

\section{Introdução}

Apesar dos gestores das pequenas e médias empresas saberem que nenhum empreendimento pode sobreviver no mercado sem estar ligado diretamente com os anseios de seus clientes, é muito comum observarmos que seus sistemas de desenvolvimento de produtos são antigos e muitas vezes entendidos como um conjunto de atividades conduzidas de forma não estruturada, baseado simplesmente na busca por soluções aos problemas técnicos de seus produtos (COOPER, 1990).

Sistemas como estes, geralmente, consideram que o mais importante é a capacidade técnica da equipe de projetistas e não levam em conta, muitas vezes, que ter um sistema estruturado pode auxiliar no processo de criação de produtos, na elaboração e condução das ideias (KAMINSKl, 2000), além de contribuir também para a busca de melhores alternativas sob o ponto de vista dos diversos processos internos da empresa.

Por outro lado, cada vez mais o mercado tem imposto aos produtos requisições de características que vêm se desenvolvendo e se atualizando de forma muito rápida, e as empresas sabem que para se manter neste mercado são obrigadas a aprender a analisar e a atender a estas necessidades a uma velocidade muitas vezes maior que o próprio surgimento delas (HUANG; MAK, 1998). 
No caso das pequenas e médias empresas, Silva (2001) pondera que, para este tipo de empresa, ser considerada como pioneira no desenvolvimento de produtos não é um dos fatores críticos de sucesso. Então, adiciona-se a esta afirmação o estudo da melhoria da eficiência da montagem e manufatura pela avaliação estruturada das condições e dos recursos produtivos disponíveis, interna e externamente, como forma de redução de custos que não estavam previstos anteriormente no projeto do produto e otimização dos prazos para lançamento de produtos. Sendo assim, o reprojeto dos produtos, apoiado por uma abordagem de engenharia reversa (ER) integrada ao projeto para manufatura e montagem (Design for Manufacturing and Assembly - DFMA), pode ser uma forma de essas empresas conseguirem lançar novos produtos com menores investimento e risco.

Uma pesquisa nas principais bases de dados científicas (Emerald, Science Direct e SciElo) revelou que este tema gerou 178 publicações em periódicos desde a década de 1980 até 2009 , como mostra a Tabela 1. A Tabela 2 mostra a distribuição dessas publicações dentro desse período para as palavras-chave product redesign, reverse engineering, design for manufacturing and assembly, design for manufacturing, design for assembly e DFMA, contidas no título do artigo. Para a base do SciElo, as mesmas palavras-chave foram empregadas também no idioma português, uma vez que nessa base se encontram os principais periódicos brasileiros da Engenharia de Produção.

Percebeu-se na análise dessas publicações que nenhuma delas abordava a integração da engenharia

Tabela 1. Publicações por palavras-chave nas principais bases de dados.

\begin{tabular}{cccc}
\hline & \multicolumn{3}{c}{ Base de dados } \\
\cline { 2 - 4 } Palavras chave & $\begin{array}{c}\text { Science } \\
\text { direct }\end{array}$ & Emerald & Scielo \\
\hline Product redesign & 4 & 2 & 0 \\
Reserve engineering & 124 & 6 & 0 \\
Design for manufacturing and... & 0 & 0 & 0 \\
Design for manufacturing & 11 & 2 & 0 \\
Design for assembly & 19 & 10 & 0 \\
DFMA & 0 & 0 & 0 \\
\hline
\end{tabular}

reversa com o DFMA no reprojeto de produtos, apenas com a prototipagem rápida. Assim, o presente trabalho pretende contribuir para a base de conhecimento preenchendo esta lacuna identificada na literatura pesquisada.

Sendo assim, o objetivo principal do presente trabalho é analisar a aplicação de um modelo para a utilização integrada do projeto para manufatura com a prototipagem rápida em uma abordagem da engenharia reversa no processo de reprojeto de produtos eletromecânicos. A partir desse objetivo principal, estabelece-se como objetivo secundário analisar os resultados da aplicação do referido modelo para a redução do tempo de produção/montagem e dos custos de manufatura/montagem no reprojeto desse produto a partir da engenharia reversa.

\section{Fundamentação teórica}

\subsection{Processo de desenvolvimento de produtos}

A geração de inovações e o lançamento de novos produtos são fundamentais para o desempenho econômico de qualquer empresa, pois exercem papéis críticos em sua competitividade.

Para Rozenfeld et al. (2006), desenvolver produtos consiste em um conjunto de atividades por meio das quais se busca, a partir das necessidades do mercado e das possibilidades e restrições tecnológicas, e considerando as estratégias competitivas e de produto da empresa, chegar a especificações de projeto de um produto e de seu processo de produção para que a manufatura seja capaz de produzi-lo.

Sobre os modelos de desenvolvimento de produtos, Ogliari (1999) cita que é possível encontrar diversos tipos disponíveis na literatura (BACK, 1983; ROSENTHAL, 1992; VINCENT, 1989; WHEELWRIGHT; CLARK, 1992; COOPER; EDGETT, 1999; PAHL et al., 2005; ROZENFELD et al., 2006; BACK et al., 2008), e a principal diferença entre eles ocorre normalmente nas denominações de suas fases, mantendo-se quase que constantes as suas sequências e conceitos.

Pahl et al. (2005) mencionam um modelo de desenvolvimentos de produtos (Figura 1) que destaca

Tabela 2. Publicações por palavras-chave entre 1980 e 2009.

\begin{tabular}{|c|c|c|c|c|c|c|}
\hline \multirow{2}{*}{ Palavras chave } & \multicolumn{6}{|c|}{ Entre 1980-2009 } \\
\hline & Anos 80 & Anos 90 & 2000-2006 & 2007 & 2008 & 2009 \\
\hline Product redesign & 1 & 2 & 2 & 0 & 1 & 0 \\
\hline Reserve engineering & 4 & 54 & 51 & 6 & 7 & 8 \\
\hline Design for manufacturing and.... & 0 & 0 & 0 & 0 & 0 & 0 \\
\hline Design for manufacturing & 1 & 6 & 3 & 1 & 1 & 1 \\
\hline Design for assembly & 8 & 13 & 4 & 0 & 2 & 2 \\
\hline DFMA & 0 & 0 & 0 & 0 & 0 & 0 \\
\hline
\end{tabular}


os aspectos importantes para a implantação da engenharia simultânea, considerando basicamente a antecipação e intersecção do início das fases para uma redução do prazo para o desenvolvimento de um novo produto e de acompanhamento de seus custos. $\mathrm{Na}$ referida figura foi focada a utilização do DFMA.

No processo de criação de um produto sob a ótica da engenharia simultânea, as atividades de cada um dos departamentos da empresa caminham, em grande parte, em paralelo. Ocorre também um permanente monitoramento do produto até o fim do seu ciclo de vida. Pahl et al. (2005) ressaltam a importância da equipe de desenvolvimento ser constituída não somente por pessoas responsáveis diretamente pelo projeto, mas também por outros setores que estejam envolvidos com o desenvolvimento de produtos, para que os aspectos ligados ao processo possam ser tratados de forma a romper as fronteiras departamentais.

Harmsen, Grunert e Bove (2000) afirmam que o processo de desenvolvimento de produtos (PDP) aumenta a cada dia sua importância em contribuir para o sucesso das organizações, consolidando-se entre os profissionais acadêmicos e empresariais como um processo crítico que necessita de aprimoramentos contínuos. Ferreira e Toledo (2001) afirmam que o PDP é uma das atividades mais importantes para ganhos em competitividade devido à crescente necessidade de se lançar produtos novos que satisfaçam às necessidades de consumidores cada vez mais exigentes.

Para Freixo (2004), é fácil perceber que decisões tomadas de forma planejada e antecipada durante as fases de um desenvolvimento do produto (sobretudo as iniciais) podem influenciar diretamente na sua dificuldade de ser fabricado. No momento de se escolher determinada alternativa de projeto, deve-se estar atento ao impacto que tal alternativa tem sobre os tempos e custos de fabricação, de operação, de manutenção e até mesmo de descarte do produto.
Além disso, decisões incorretas tomadas no início do desenvolvimento do produto podem ser muito difíceis de serem revertidas. lsso vale também para o caso dos reprojetos de produtos.

0 modelo de desenvolvimento de produtos, em uma última análise, irá depender do tipo de empresa e da estratégia que ela utiliza. Griffin e Page (1996) citam seis tipologias para a estratégia de projeto em relação ao desenvolvimento de novos produtos. São elas: novos para o mundo; novos para a organização; aditivos a linhas existentes de produto; melhorias/ revisões em produtos existentes; reposicionamentos; reduções de custo.

Visto que, em geral, a condição das pequenas e médias empresas (PMEs) não é necessariamente a de pioneirismo e que suas estratégias de desenvolvimento de produtos não são necessariamente ofensivas (SILVA, 2001), devido à necessidade de grandes investimentos em pesquisa e desenvolvimento tecnológico, muitas vezes, este entendimento e uma revisão na estratégia passam a ser o ponto-chave para a redução de custos ou, até mesmo, a possibilidade única de desenvolvimento de novos produtos de uma forma estruturada e com maiores chances de sucesso. Dessa forma, a abordagem da engenharia reversa para o processo de desenvolvimento de produtos passa a ser uma possível solução para a inovação nessas empresas.

\subsection{Engenharia reversa}

Entre as principais técnicas existentes para o desenvolvimento ou reprojeto de produtos, encontra-se a Engenharia Reversa (ER), um tema pouco abordado e difundido nos países geradores de tecnologia por ser frequentemente confundido com simples cópia de produtos.

Segundo Kim e Nelson (2005), países com industrialização recente recorreram, principalmente

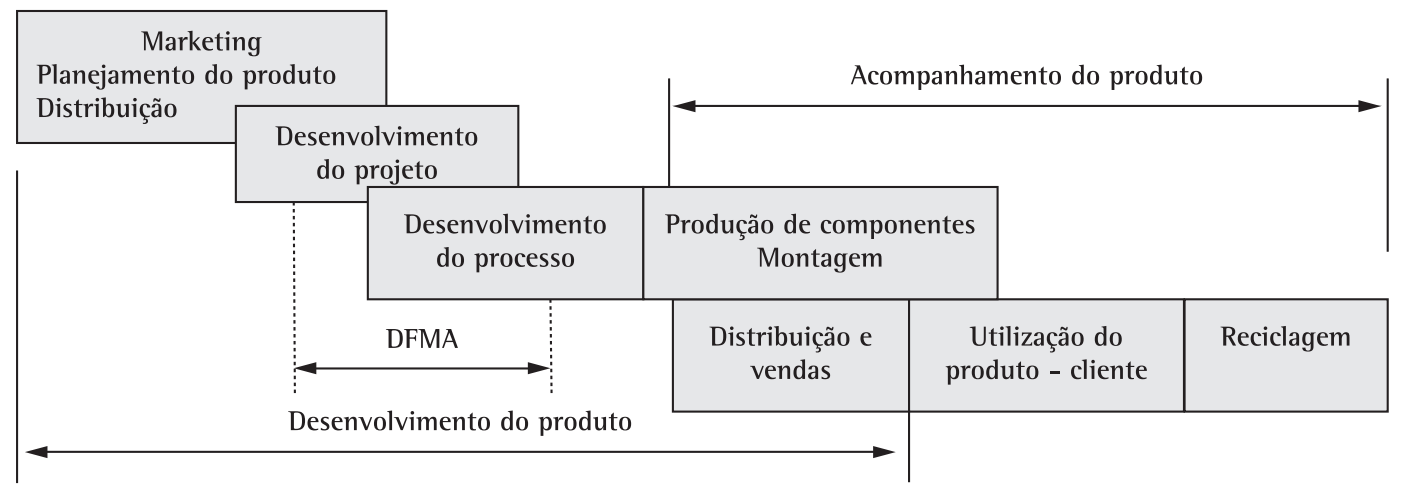

Figura 1. Modelo de referência para o PDP. Fonte: Pahl et al. (2005). 
nas décadas de 1960 e 1970, à engenharia reversa. Zhu, liang e Xu (2005) afirmam que o processo de aquisição de tecnologia da China segue, geralmente, a seguinte linha: aquisição de linhas de manufatura e técnicas de países desenvolvidos, modificação do processo e identificação das partes e componentes, alcançar o desenvolvimento do produto através da ER e, por fim, otimizar os produtos. 0 processo de inovação da Coreia do Sul é por meio da ER, esperando os países desenvolvidos gerarem novas tecnologias e mercados para, aí sim, desenvolver seus produtos (HOBDAY, RUSH; BESSANT, 2004).

Esta técnica, no entanto, quando inserida em uma metodologia para implementação de melhorias em produtos, toma como ponto de partida as especificações técnicas de um produto já lançado no mercado, objetivando o aperfeiçoamento e não a simples cópia do produto já existente (DIAS, 1998). No que tange ao desenvolvimento ou adaptação de um produto, o ponto crítico para a ER é a formulação de especificações desse novo produto, sendo, para tanto, necessárias atividades de pesquisa e desenvolvimento. A elaboração do projeto do processo produtivo também apresenta uma complexidade que demanda conhecimentos técnicos.

Ingle (1994) define engenharia reversa (ER) como uma atividade que trabalha com um produto existente (um software, um componente mecânico, um produto eletromecânico etc.) e tenta entender como este produto funciona e o que ele faz exatamente (todas as suas propriedades em quaisquer circunstâncias). Utiliza-se a engenharia reversa quando se deseja trocar ou modificar uma peça (ou um software) por outra, com as mesmas características, mas não se têm em mãos todas as informações sobre essa peça. Basicamente, consiste na tentativa planejada e organizada de obter tecnologia (produto e processo) através da análise de produtos de referência (os melhores daquele segmento).

A principal aplicação da ER é no reprojeto e aperfeiçoamento de peças já existentes, nas quais sejam desejadas melhorias, tais como redução de custo ou mesmo inclusão de novas características ao produto. Além disso, um projeto de ER permite, através da construção de peças de reposição, fora de linha ou de difícil acesso, manter equipamentos obsoletos em funcionamento (MURY, 2000).

A sistemática de Otto e Wood (1998) para a engenharia reversa oferece três tipos de resultados: o reprojeto paramétrico, para otimizar parâmetros do produto; o reprojeto adaptativo, para desenvolvimento e teste de novos subsistemas; e o reprojeto original, para o desenvolvimento de novos produtos. Em nenhum momento esses autores tratam da ER como simples cópia. lsso mostra que a ER pode ser utilizada como um processo de inovação, tal como em Mello et al. (2010), uma vez que, se esse cuidado não for tomado, a empresa pode ter problemas com a propriedade intelectual do produto adotado como referência.

Ingle (1994) chega a propor, com certa dose de exagero, a ER como um processo de desenvolvimento de produtos, como mostra a Figura 2.

0 Quadro 1 descreve de forma resumida as etapas para a ER adotadas para a presente pesquisa.

Apesar de muito citado na literatura, o modelo de Ingle (1994) não contempla a integração do projeto para manufatura e montagem com a prototipagem rápida em uma abordagem de engenharia reversa para o reprojeto de produtos. Essa é uma contribuição científica que o presente trabalho busca oferecer.

Outra abordagem que, de forma integrada com a ER, pode auxiliar a análise de reprojeto de produtos é o projeto para manufatura e montagem (DFMA).

\subsection{Projeto para manufatura e montagem (DFMA)}

0 DFMA visa que o projeto de produto e o planejamento da produção aconteçam simultaneamente, a partir de um conjunto de princípios. Já no reprojeto, o DFMA ajuda a adequar
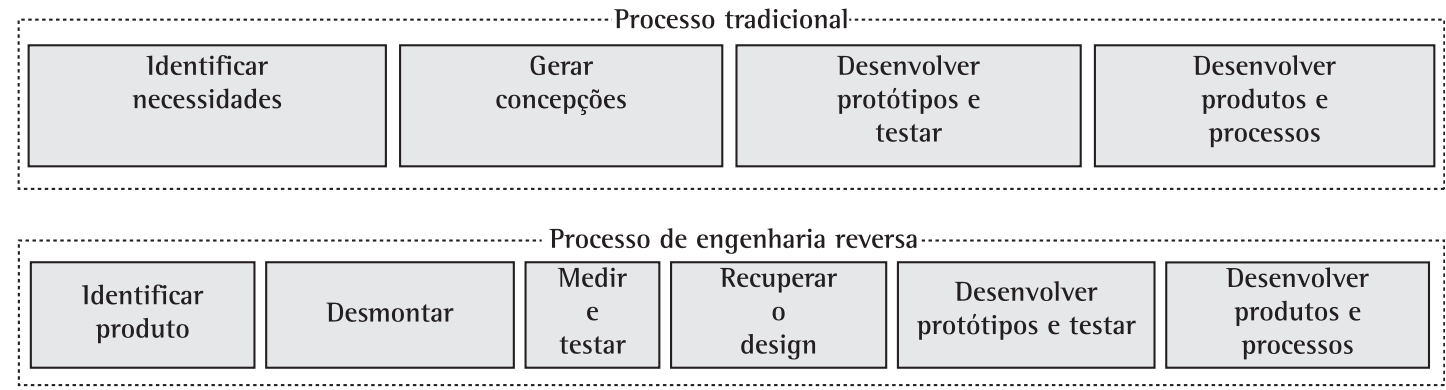

Figura 2. Comparação dos processos de desenvolvimento de produto tradicional e através da ER. Fonte: Ingle (1994). 
Quadro 1. Etapas da engenharia reversa.

\begin{tabular}{|c|c|}
\hline Etapa & Resultados \\
\hline $\begin{array}{l}\text { 1dentificar produtos de excelência } \\
\text { sobre os quais se deseja proceder } \\
\text { a Engenharia Reversa. }\end{array}$ & $\begin{array}{l}\text { Definição do produto de } \\
\text { referência adotado. }\end{array}$ \\
\hline $\begin{array}{l}\text { 1dentificar os parâmetros a serem } \\
\text { analisados. }\end{array}$ & Parâmetros de análise. \\
\hline $\begin{array}{l}\text { Analisar o produto: simples } \\
\text { análise visual, submeter o produto } \\
\text { a testes e ensaios, desmontar o } \\
\text { produto com o mapeamento dos } \\
\text { seus componentes. }\end{array}$ & $\begin{array}{l}\text { Diferenças construtivas visuais; } \\
\text { resultados dos testes e ensaios; } \\
\text { Tabela de componentes. }\end{array}$ \\
\hline $\begin{array}{l}\text { Selecionar e analisar os } \\
\text { componentes quanto às } \\
\text { características dos materiais e } \\
\text { dimensionais. }\end{array}$ & $\begin{array}{l}\text { Desenhos com especificações } \\
\text { técnicas e dimensionais dos } \\
\text { componentes selecionados. }\end{array}$ \\
\hline $\begin{array}{l}\text { Elaborar Tabelas comparativas } \\
\text { entre os produtos já existentes e } \\
\text { produto que se quer implantar. }\end{array}$ & $\begin{array}{l}\text { Identificação de melhorias no } \\
\text { produto em estudo. }\end{array}$ \\
\hline Gerar novo produto. & $\begin{array}{l}\text { Produto aperfeiçoado } \\
\text { (semelhante ao comercializado, } \\
\text { com melhorias). }\end{array}$ \\
\hline $\begin{array}{l}\text { Avaliar os resultados e propor } \\
\text { aperfeiçoamentos. }\end{array}$ & $\begin{array}{l}\text { Aprendizado (geração de } \\
\text { conhecimento). }\end{array}$ \\
\hline
\end{tabular}

Fonte: Adaptado de Ingle (1994).

o produto da melhor maneira às características da produção e montagem, procurando melhorar a qualidade e reduzir o tempo de manufatura e montagem (DUFOUR, 1996).

0 uso dos princípios do DFMA para melhorar o produto só inicia na fase de reprojeto preliminar. Contudo, para o DFMA, esta fase é tão importante como a de detalhamento, na qual realmente as melhorias tornam-se efetivas, avaliando a estrutura, a geometria global das partes e os materiais.

A abordagem desses princípios no reprojeto está baseada nas consequências das escolhas feitas pelos projetistas durante o processo de projeto e tem a finalidade de melhorar o produto original em relação à produção e montagem (DUFOUR, 1996).

Para isto, devem ser estabelecidos os objetivos do reprojeto em relação aos princípios do DFMA, tais como: identificar os problemas que afetam a produção e montagem de um produto dentro de determinadas especificações (concepção original); enfocar o reprojeto dos componentes visando a uma fabricação e montagem mais simples, reduzindo o número de partes e facilitando a manipulação e inserção destas, além de eliminar aquelas que encarecem o produto; integrar os projetos do processo de fabricação e montagem com o reprojeto do produto, para assegurar o melhor atendimento dos requisitos.

Para reprojetar um produto enfocando sua manufatura e montagem, o DFMA deve ser discutido em vários níveis, como recomenda Andreasen (1991): nível da empresa, em que são feitas as escolhas estratégicas de tecnologia e materiais que devem ser adaptados à tecnologia estratégica do produto; nível de classificação do produto, em que são decididas as famílias, reutilização e modulação do produto, assim como os processos e equipamentos; nível de estrutura do produto, em que especialmente a estrutura de montagem influencia a produção e a sequência da montagem; nível do componente, em que é feita uma escolha detalhada da cadeia de processo para cada parte e na qual o projeto detalhado de cada uma, deve assegurar uma produção eficiente e sem problemas.

Em função de um ou mais destes níveis é que surgirão as melhorias do produto. 0 projeto ou concepção original é reavaliado e os métodos de montagem e processos de manufatura mais adequados são escolhidos. Neste trabalho, optou-se por analisar o reprojeto do produto selecionado no nível de estrutura de produto e de seus componentes.

Os projetistas têm que lidar com uma grande quantidade de informações que, ao serem reavaliadas, ajudarão a determinar as mudanças que o produto poderá sofrer. Estas informações incluem todos os dados que foram utilizados pelos projetistas para definir as partes e montagens. Elas podem ser subdivididas em informações sobre o projeto original, materiais e processos utilizados e serão empregadas no reprojeto para avaliar e modificar o projeto ou produto, como forma de melhorar a sua capacidade de manufatura (MYRUP, 1991).

Usando essas informações, os projetistas podem melhorar a qualidade do produto e reduzir os tempos e custos de manufatura. Estas informações são úteis para avaliar a concepção inicial em relação às virtudes universais do DFMA (custo, tempo, qualidade, eficiência e flexibilidade do sistema produtivo), e determinar falhas no produto, visando a sua melhoria (MATOUSEK, 1957).

Outro fator importante no reprojeto de um produto é verificar e complementar os requisitos de manufatura e montagem, visto que o produto deve ser reprojetado para satisfazer seus requisitos funcionais (DUFOUR, 1996).

Segundo Stephenson e Wallace (1995), os requisitos da concepção original devem ser reavaliados para estabelecer os novos requisitos de qualidade DFMA, considerando sempre os seguintes princípios básicos do projeto para manufatura (Design for Manufacturing - DFM) e do projeto para montagem (Design for Assembly - DFA): simplicidade (diminuir o número de partes, sequência de manufatura mais curta etc.); materiais e componentes padronizados; liberar tolerâncias (evitar tolerâncias muito justas que implicam em altos custos); uso de materiais mais processáveis; reduzir operações secundárias; utilizar 
características especiais de processo (tirar vantagem das capacidades especiais dos processos de manufatura, eliminando operações onerosas e desnecessárias); evitar limitações no processo.

Além do DFMA, a prototipagem rápida (PR) é uma técnica que pode contribuir na redução do tempo de reprojeto de produtos, auxiliando na produção de protótipos rápidos para a engenharia reversa.

\subsection{Prototipagem rápida}

A prototipagem rápida ( $\mathrm{PR}$ ) é um processo de fabricação inovador desenvolvido nas últimas duas décadas. Ela visa produzir protótipos de forma relativamente rápida para inspeção visual, avaliação ergonômica, análise de forma/dimensional e como padrão mestre para a produção de ferramentas para auxiliar na redução de tempo do processo de desenvolvimento de produtos (CHOI; CHAN, 2004). A PR permite aos projetistas criar rapidamente protótipos concretos a partir de seus projetos, ao invés de Figuras bidimensionais, possibilitando um auxílio visual excelente durante a discussão prévia do projeto com colaboradores ou clientes.

Atualmente existe um grande número de tecnologias de prototipagem rápida disponíveis no mercado. Entretanto, sete delas se destacam: estereolitografia (SLA), sinterização seletiva a laser (SLS), manufatura de objetos em lâminas (LOM), modelagem por deposição de material fundido (FDM) e impressão tridimensional ( $3 D$ Printing) (CHEN, 2000).

A presente pesquisa se concentrou na tecnologia de modelagem por deposição de material fundido (Fused Deposition Modelling - FDM), por ser aquela que oferece equipamentos de menor custo (KOCHAN,
2000), ou seja, ao alcance das pequenas e médias empresas e das instituições de pesquisa.

A FDM se baseia na deposição, sobre uma plataforma, de camadas resultantes do aquecimento e amolecimento de filamentos do material plástico destinado à confecção do modelo. Simultaneamente, outros fios amolecidos vão formando suportes para as superfícies livremente suspensas do modelo, a fim de que elas possam ser construídas. Os arames destinados ao modelo são, geralmente, de ABS (Acrylonitrile Butadiene Styrene), enquanto os destinados aos suportes são uma mistura de ABS e cal.

A partir do protótipo gerado, a equipe de projeto pode analisar o produto adotado como referência, testar suas especificações, testar situações de manufatura ou montagem, propor alterações dimensionais ou construtivas e estabelecer as possíveis melhorias a serem feitas no produto final a ser desenvolvido.

\subsection{Integração da ER com o DFMA e a PR}

Souza (2007) propôs uma integração entre a ER, o DFMA e a PR, dada pela Figura 3, a partir dos modelos de Pahl et al. (2005) e lngle (1994).

Analisando o trabalho proposto por Ingle (1994), Souza (2007) observou que existia uma grande deficiência nas considerações relativas às necessidades da manufatura e montagem. Desta forma, o modelo proposto por ele considera que os fundamentos do DFMA sejam incluídos durante as análises de engenharia reversa, fazendo com isto um complemento ao proposto por Ingle (1994). A avaliação destas necessidades o levou a propor um modelo composto por oito etapas, conforme mostra a Figura 4.

0 Quadro 2 explica cada uma das oito etapas do modelo proposto por Souza (2007).

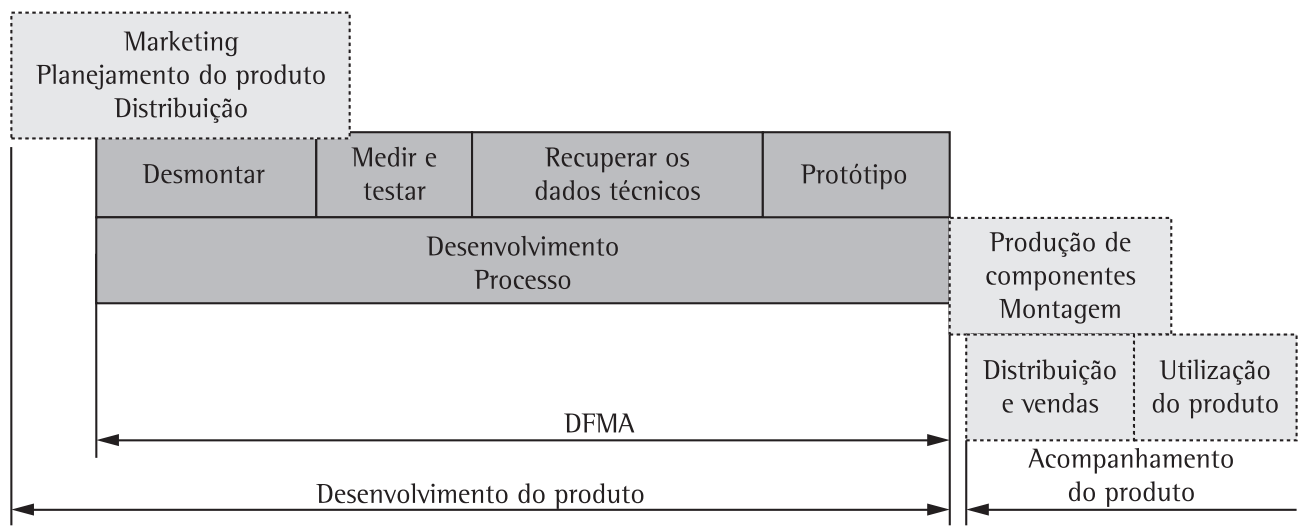

Figura 3. Integração da ER com o DFMA e a PR. Fonte: Souza (2007). 


\section{Método de pesquisa}

0 método de pesquisa empregado foi a pesquisaação. Segundo Thiollent (2007), a pesquisa-ação é um tipo de pesquisa social com base empírica que é concebida e realizada em estreita associação com uma ação ou com a resolução de um problema coletivo e no qual os pesquisadores e os participantes representativos da situação ou do problema estão envolvidos de modo cooperativo ou participativo.

0 processo de pesquisa-ação adotado foi aquele proposto por Coughlan e Coghlan (2002), que contempla ciclos de planejamento (contexto e problema), coleta de dados, revisão dos dados, análise dos dados, planejamento de ações, implementação e avaliação, como mostra a Figura 5.

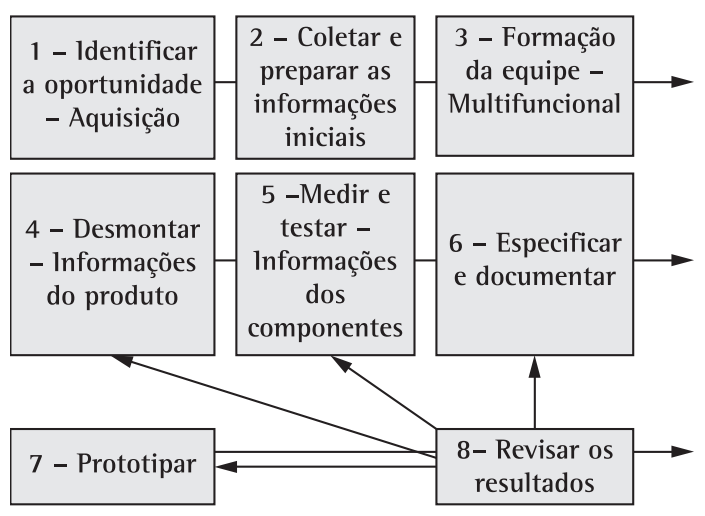

Figura 4. Modelo proposto para integração da ER com o DFMA. Fonte: Souza (2007).
Neste presente trabalho, a empresa selecionada como objeto de estudo produz um dispositivo eletromecânico para controle de tempos de funcionamento de máquinas e equipamentos, denominado de horímetro.

A fase do estudo preliminar no presente trabalho compreendeu reuniões de planejamento, realizadas com o intuito de identificar os principais problemas do referido produto, que foi projetado há mais de 30 anos. Assim, o seguinte propósito foi estabelecido: reprojetar o horímetro, a partir do modelo proposto por Souza (2007), para atender às necessidades atuais dos clientes e também reduzir custos, uma vez que a concorrência começa a ameaçar a participação de mercado da empresa.

Na segunda fase, ou seja, o ciclo de condução constituído por seis passos (Figura 5) foi repetido quatro vezes, como mostra o Quadro 3.

$\mathrm{Na}$ terceira (meta) fase, denominada de monitoramento (Figura 5), foi realizada a verificação de cada um dos seis passos anteriores, no sentido de identificar qual é o aprendizado gerado na condução da pesquisa-ação. 0 monitoramento gerou diversas lições aprendidas que foram registradas na base de dados da empresa para utilização em projetos futuros.

\section{Descrição da pesquisa-ação realizada}

A empresa selecionada para a pesquisa foi a Ômega Micromecânica, uma empresa localizada em ltajubá, ao sul de Minas Gerais, que está entre as três maiores fornecedoras nacionais de horímetros para os fabricantes de máquinas agrícolas e para o mercado de reposição.

Quadro 2. Etapas do modelo proposto por Souza (2007).

\begin{tabular}{|c|c|}
\hline Etapa & Significado \\
\hline Identificar a oportunidade & $\begin{array}{l}\text { ldentificar e reconhecer a posição da empresa no mercado em que atua e identificar qual é o produto a ser } \\
\text { considerado como referência. }\end{array}$ \\
\hline $\begin{array}{l}\text { Coletar e preparar as } \\
\text { informações iniciais }\end{array}$ & $\begin{array}{l}\text { Obter um levantamento inicial do mercado: não somente do produto de referência, mas de todos que podem } \\
\text { compor um nível de conhecimento sobre a classe de produtos que se está analisando. Coletar e dispor todas } \\
\text { as informações que possam contribuir para a aplicação da engenharia reversa com a aplicação de técnicas de } \\
\text { projeto para a manufatura e montagem (DFMA). }\end{array}$ \\
\hline $\begin{array}{l}\text { Formação da equipe } \\
\text { multifuncional }\end{array}$ & $\begin{array}{l}\text { A equipe multidisciplinar de desenvolvimento deveria conter elementos que detêm o conhecimento teórico e } \\
\text { prático sobre todos os detalhes e características do produto produzido pela empresa, e estes conhecimentos } \\
\text { devem estar disponiveis para toda a equipe, para que nenhuma oportunidade passe despercebida. }\end{array}$ \\
\hline $\begin{array}{l}\text { Desmontar (coletar } \\
\text { informações do produto) }\end{array}$ & $\begin{array}{l}\text { Analisar tecnicamente os sistemas de forma sistemática e as funções envolvidas em cada um dos componentes } \\
\text { e subsistemas do produto de referência (ou dos produtos), para se disponibilizar informações que serão } \\
\text { analisadas mais detalhadamente na fase seguinte. É necessário ter uma preocupação principal na interação } \\
\text { entre os componentes, com uma visão muito focada em detalhes técnicos (tolerâncias, fixações, ajustes etc.). }\end{array}$ \\
\hline $\begin{array}{l}\text { Medir e testar (coletar } \\
\text { informações dos } \\
\text { componentes) }\end{array}$ & $\begin{array}{l}\text { As ações realizadas nesta fase estão ligadas à medição e realização de testes dos componentes do produto } \\
\text { de referência, procurando esclarecer as dúvidas relativas às tecnologias de produto e de processo empregadas } \\
\text { no conjunto e nos componentes. }\end{array}$ \\
\hline Especificar e documentar & $\begin{array}{l}\text { Documentar tecnicamente as informações levantadas na fase anterior e especificar novas que foram deixadas } \\
\text { para esta fase por pessoas mais ligadas diretamente aos detalhamentos de componentes e processos de produção. }\end{array}$ \\
\hline Prototipar & $\begin{array}{l}\text { Utilizar os protótipos (rápidos), dentro do ambiente da engenharia reversa, para auxílio como fonte de entrada } \\
\text { de informações nas análises de DFMA. }\end{array}$ \\
\hline Revisar os resultados & $\begin{array}{l}\text { Realizar um gerenciamento sobre todas as fases anteriores e de como o processo está caminhando em relação } \\
\text { a prazos e custos. Esta fase é conduzida pelo pesquisador coordenador do projeto e deve ser utilizada como } \\
\text { centralizadora das informações. }\end{array}$ \\
\hline
\end{tabular}


O horímetro (uma espécie de cronômetro) é um dispositivo eletromecânico para o controle dos tempos de trabalho de máquinas, equipamentos e motores estacionários. Dentre suas principais aplicações estão: tratores e máquinas agrícolas em geral, empilhadeiras, motores marítimos, máquinas industriais e semelhantes. Esse produto foi escolhido por ser um dos maiores faturamentos da empresa, mas que atualmente encontra-se com sua participação no mercado ameaçada por seus concorrentes que possuem produtos mais avançados tecnologicamente. Dessa forma, a empresa necessita reprojetar seu produto, visando reduzir seu custo-meta para, no mínimo, manter sua atual participação no mercado.

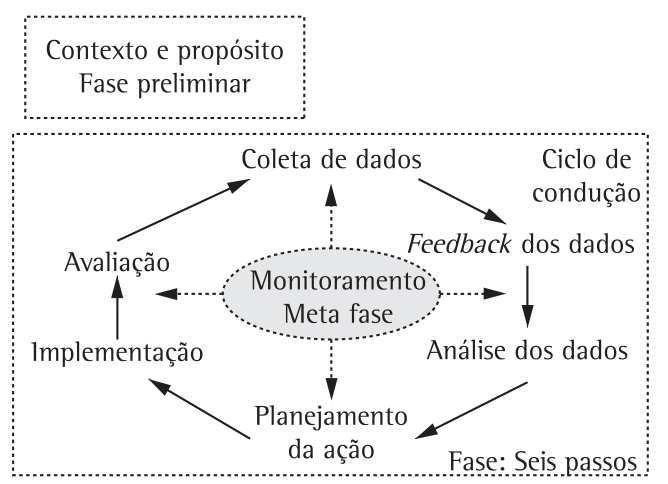

Figura 5. Abordagem da pesquisa-ação. Fonte: Miguel (2009).
A realização da pesquisa seguiu as etapas do modelo de integração proposto por Souza (2007), citadas no Quadro 2.

\subsection{Identificação da oportunidade e aquisições do produto considerado como referência no mercado}

Para o reprojeto do horímetro por meio da engenharia reversa, foi selecionado como produto de referência um horímetro de uma empresa europeia com representação comercial no Brasil. A decisão pela escolha foi tomada em conjunto com o departamento comercial da empresa objeto de estudo. Este produto foi considerado de referência por ser um dos líderes de venda nesse mercado.

0 tipo de reprojeto adotado, conforme Otto e Wood (1998), foi o adaptativo, pela necessidade de reprojeto de novos subsistemas para o horímetro da empresa objeto de estudo.

A aquisição do produto de referência foi possível graças ao apoio financeiro oferecido pela FAPEMIG, junto ao edital "Programa Jovens Doutores", processo EDT-538/07, que propiciou um recurso de, aproximadamente, $R \$ 25.000,00$ para a realização da pesquisa. Ressalta-se que o produto fruto desta pesquisa não infringiu qualquer patente ou propriedade industrial, uma vez que as soluções para os subsistemas

Quadro 3. Resumo dos ciclos da pesquisa-ação realizada.

\begin{tabular}{|c|c|c|c|c|}
\hline Ciclo & Período & Atividades executadas & Reflexão/avaliação & Plano de ação \\
\hline 1 & $\begin{array}{l}\text { Agosto de } \\
2007 \text { a } \\
\text { Outubro } \\
\text { de } 2007\end{array}$ & $\begin{array}{l}\text { Busca por produto de referência no } \\
\text { mercado. } \\
\text { Levantamento de dados do modelo atual do } \\
\text { horímetro. }\end{array}$ & $\begin{array}{l}\text { Foram identificados três } \\
\text { fabricantes de horímetros. } \\
\text { Os desenhos do horímetro } \\
\text { apresentavam falhas, como } \\
\text { cotas faltantes. } \\
\text { Não havia um levantamento de } \\
\text { tempos do processo. }\end{array}$ & $\begin{array}{l}\text { Selecionar o melhor produto entre os } \\
\text { três identificados. } \\
\text { Refazer desenhos do horimetro em } \\
\text { software paramétrico 3D utilizando } \\
\text { amostras de cada componente. } \\
\text { Mapear processos de montagem e } \\
\text { levantar tempos do processo. }\end{array}$ \\
\hline 2 & $\begin{array}{l}\text { Novembro } \\
\text { de } 2007 \text { a } \\
\text { Julho de } \\
2008\end{array}$ & $\begin{array}{l}\text { Definição do produto de referência, com } \\
\text { base em análise do grupo de pesquisa. } \\
\text { Redesenho do horímetro atual em software } \\
\text { 3D para facilitar o reprojeto do conceito. } \\
\text { Levantamento dos tempos de cada processo } \\
\text { e custos de cada conjunto e componente. }\end{array}$ & $\begin{array}{l}\text { Foram avaliados os aspectos } \\
\text { construtivos, tecnologia e } \\
\text { realizados testes no horimetro } \\
\text { de referência. } \\
\text { Desmontagem do horímetro } \\
\text { de referência para avaliação de } \\
\text { cada conjunto/componente. }\end{array}$ & $\begin{array}{l}\text { Acompanhar processo de montagem do } \\
\text { horímetro. } \\
\text { Levantar problemas de montagem e } \\
\text { fabricação in loco (observar e entrevistar } \\
\text { montadoras). } \\
\text { Analisar com a equipe de projeto as } \\
\text { propostas de melhoria do produto. } \\
\text { Desenvolver princípios de solução para } \\
\text { o produto (em 3D). }\end{array}$ \\
\hline 3 & $\begin{array}{l}\text { Agosto de } \\
2008 \text { a } \\
\text { Dezembro } \\
\text { de } 2008\end{array}$ & $\begin{array}{l}\text { Elaboração de mapa do processo de } \\
\text { montagem com tempos padrão de cada etapa. } \\
\text { Definição de conjuntos específicos do } \\
\text { horímetro para reprojeto inicial. } \\
\text { Elaboração de relatório de problemas de } \\
\text { montagem. } \\
\text { Desenvolvimento dos primeiros princípios de } \\
\text { solução para reprojeto. }\end{array}$ & $\begin{array}{l}\text { Princípios de solução analisados } \\
\text { e selecionados pela equipe de } \\
\text { projeto. } \\
\text { Verificação da necessidade de } \\
\text { ajustes em algumas propostas } \\
\text { de reprojeto dos componentes. } \\
\text { Novas sugestões de melhorias. }\end{array}$ & $\begin{array}{l}\text { Refazer desenhos tridimensionais para } \\
\text { apresentação para equipe de projeto. } \\
\text { Prototipar peças reprojetadas para } \\
\text { análise dimensional e de montagem dos } \\
\text { componentes. } \\
\text { Montagem de um protótipo híbrido } \\
\text { para simulação de testes de campo } \\
\text { (principalmente, vibração). }\end{array}$ \\
\hline 4 & $\begin{array}{l}\text { Janeiro } \\
\text { de } 2009 \\
\text { a Abril de } \\
2009\end{array}$ & $\begin{array}{l}\text { Apresentação dos desenhos 3D do horímetro } \\
\text { reprojetado. } \\
\text { Apresentação das peças prototipadas para } \\
\text { análise da equipe de pesquisa. } \\
\text { Elaboração de protótipo híbrido para testes. }\end{array}$ & $\begin{array}{l}\text { A equipe de projeto aprovou os } \\
\text { componentes reprojetados. Uma } \\
\text { estimativa mostrou que pode } \\
\text { ocorrer redução no custo-meta } \\
\text { do produto, além de outras } \\
\text { melhorias. }\end{array}$ & $\begin{array}{l}\text { Realizar testes no protótipo híbrido } \\
\text { (construídos com peças em produção } \\
\text { e peças reprojetadas prototipadas) } \\
\text { para verificar a viabilidade técnica do } \\
\text { produto reprojetado. }\end{array}$ \\
\hline
\end{tabular}


gerados foram diferentes daquelas observadas no produto de referência.

\subsection{Coletar e preparar dados iniciais}

Esta fase é a primeira com características técnicas envolvidas. Foi realizado um levantamento inicial do mercado de instrumentos de medida para painéis de máquinas e equipamentos agrícolas e motores estacionários.

Foram coletadas e dispostas todas as informações que poderiam contribuir para a integração da engenharia reversa com o DFMA e a PR: elaboração da estrutura do produto (definição dos conjuntos e seus componentes), mapeamento e cronoanálise do processo de montagem do horímetro, definição do tempo padrão de cada etapa do processo de montagem e levantamento dos custos do processo de montagem do produto.

Uma série de entrevistas não estruturadas foi realizada com as funcionárias encarregadas da montagem para identificar suas principais dificuldades no processo de montagem do horímetro. 0 Quadro 4 mostra algumas das dificuldades identificadas.

Quadro 4. Diagnóstico das partes consideradas no reprojeto.

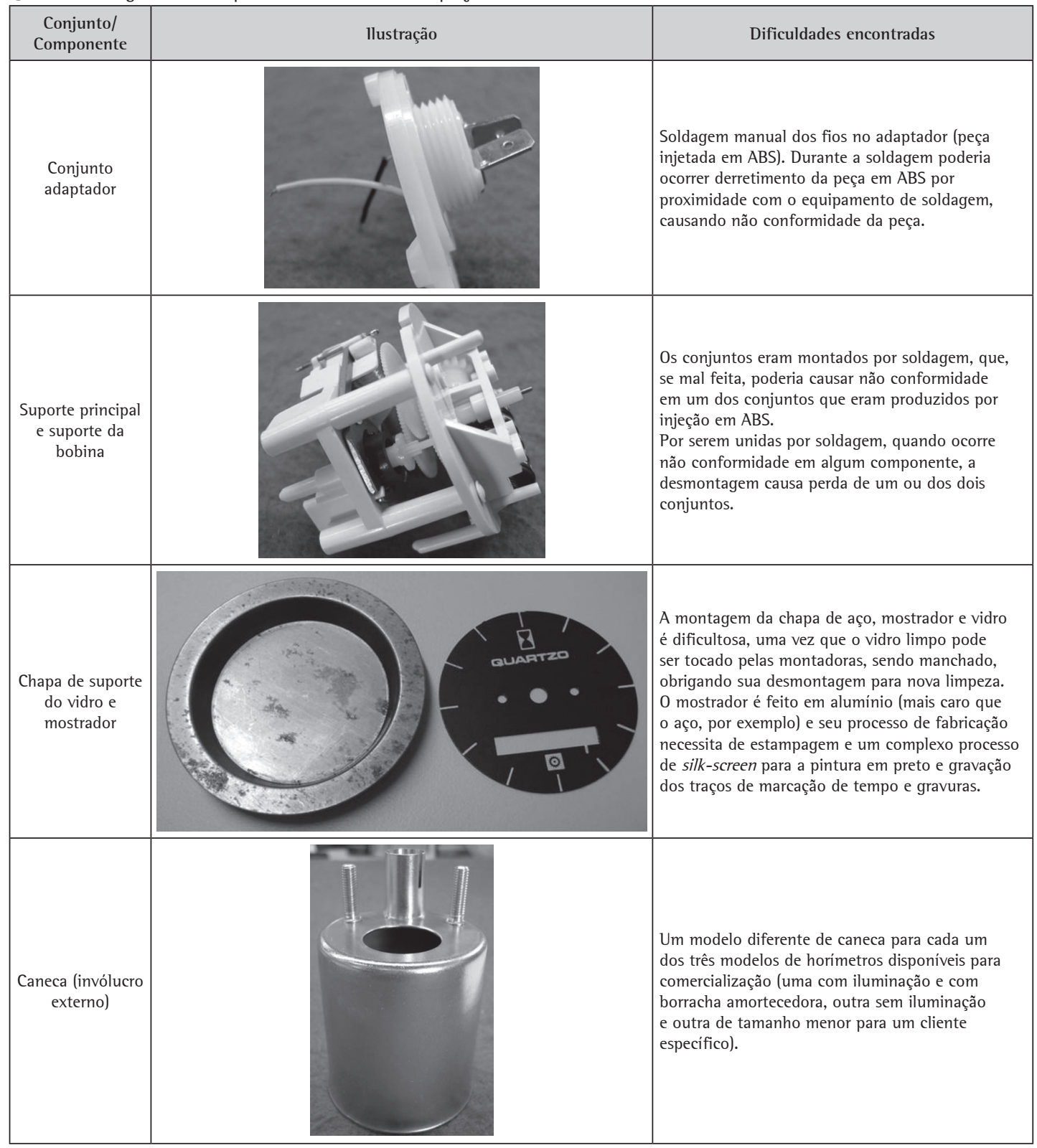




\subsection{Formação da equipe}

0 Quadro 5 apresenta o grupo de pesquisa formado para o desenvolvimento do presente trabalho.

\subsection{Desmontar (informações sobre o produto)}

0 horimetro estudado possui 11 conjuntos e 87 componentes ao todo. Para o reprojeto deste produto por meio da engenharia reversa, o produto de referência foi totalmente desmontado para que o grupo de pesquisa pudesse analisar seus aspectos construtivos, materiais, (provável) processo de montagem, número de componentes etc.

A análise do produto de referência revelou diversas informações técnicas que poderiam ser úteis na comparação dos componentes equivalentes do horímetro estudado. 0 Quadro 6 apresenta uma comparação entre alguns desses componentes.

\subsection{Medir e testar (informações sobre os componentes)}

Após ser desmontado, o produto adotado como referência foi remontado e levado ao laboratório da empresa para testes de burn-in, entre outros. Foram adquiridos alguns modelos de painéis de tratores para teste de instalação do horímetro reprojetado.

Após essas análises, diversas reuniões entre os integrantes do grupo de pesquisa foram realizadas no sentido de se tentar incorporar soluções ao produto atual que, baseadas nas análises do produto de referência e nos princípios do DFMA, pudessem melhorar o seu processo de montagem e reduzir o seu custo-meta.

\subsection{Especificar e documentar}

A empresa estudada produzia três modelos de horímetros, sendo um para atender um cliente específico com uma caneca de tamanho menor (a caneca é o invólucro que protege todos os outros componentes e que ajuda na fixação do horímetro no painel do trator), um com iluminação e com borracha amortecedora e outro sem iluminação (para esses dois últimos, as canecas são diferenciadas para comportar, ou não, no seu fundo o conjunto soquete para a lâmpada). Uma das sugestões do grupo foi propor também uma caneca única para o horímetro a ser reprojetado. Outras análises similares foram realizadas para outros conjuntos e componentes. Todas as sugestões dadas pelo grupo de pesquisa foram documentadas e as mudanças nos componentes ou conjuntos especificadas.

0 Quadro 7 apresenta algumas das soluções propostas para a empresa como resultado do reprojeto do produto.

\subsection{Prototipar}

Para a apresentação das soluções propostas, foram construídos protótipos rápidos dos componentes para facilitar o entendimento por parte de todos os envolvidos e propiciar a realização de testes (dimensionais e visuais), a fim de se construir um protótipo híbrido (com peças prototipadas e peças utilizadas em modelos de horímetros em produção) para futuros testes mais esclarecedores (por exemplo, vibração).

Os protótipos foram construídos na máquina de prototipagem rápida Stratasys Dimension SST 768 do Laboratório de Inovação de Produtos (LIP) do Núcleo de Otimização da Manufatura e da Tecnologia da Inovação (NOMATI) da Universidade Federal de Itajubá (UNIFEI). Para cada conjunto modificado foi construído seu protótipo correspondente. Posteriormente, foi construído um protótipo híbrido para discussão pela equipe de pesquisa e realização de testes. A Figura 6 mostra o protótipo híbrido construído para a realização dos testes.
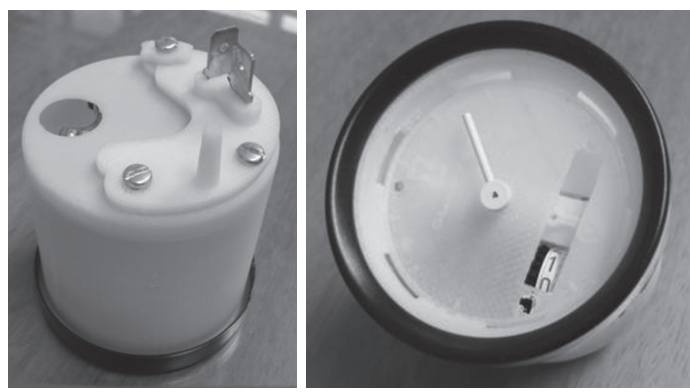

Figura 6. Protótipo híbrido.

Quadro 5. Equipe de pesquisa

\begin{tabular}{|l|l|l|}
\hline \multicolumn{1}{|c|}{ Membro } & \multicolumn{1}{c|}{ Formação } & \multicolumn{1}{c|}{ Experiência } \\
\hline Pesquisador coordenador & Doutor em Engenharia de Produção & $\begin{array}{l}\text { Pesquisas em processos de desenvolvimento de produtos } \\
\text { há mais de quatro anos. }\end{array}$ \\
\hline Pesquisador & Doutor em Engenharia de Produção & Especialista em projetos mecânicos. \\
\hline Bolsistas de iniciação científica (dois) & Graduandos em Engenharia Mecânica & Desenhos de projetos mecânicos. \\
\hline Gerente de vendas & Administrador de empresas & Mais de cinco anos na comercialização do horímetro. \\
\hline Gerente da qualidade & Técnico & Mais de 20 anos na produção do horímetro. \\
\hline
\end{tabular}


Quadro 6. Comparação de componentes entre horímetro estudado e de referência.

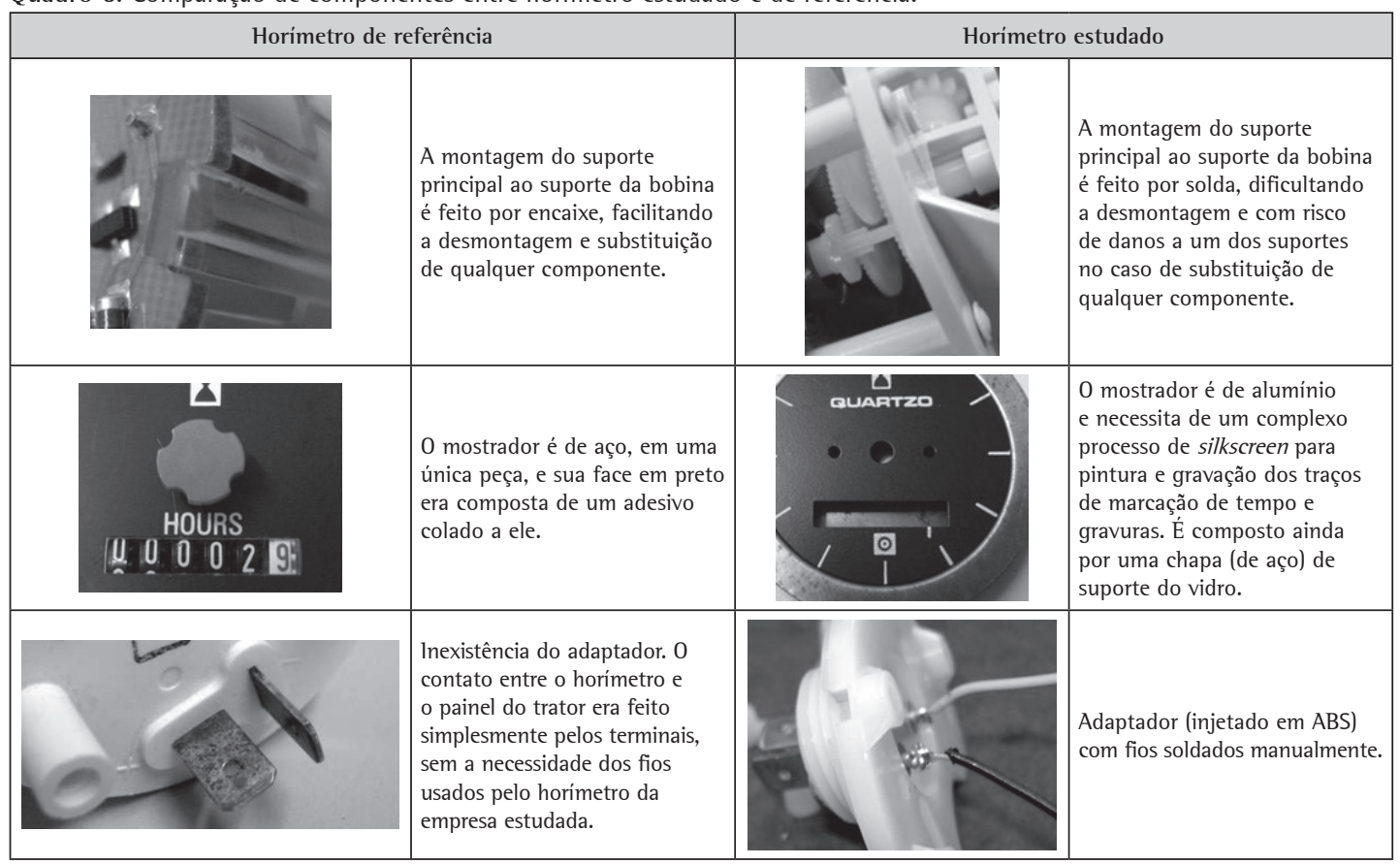

\subsection{Rever os resultados}

Os testes realizados propiciaram oportunidades de melhorias nos componentes reprojetados. Por exemplo, o teste de vibração mostrou que os conjuntos unidos por encaixe apresentavam desempenho similar ao do produto original (união por solda). Isso deu segurança aos proprietários da empresa de que as propostas de alterações poderiam ser levadas adiante, minimizando os riscos de insucesso. Essas informações foram registradas na base de dados da empresa, na forma de lições aprendidas.

\section{Análise dos resultados}

A proposta das soluções, dentre as quais aquelas apresentadas no Quadro 7, propiciou diversos benefícios para o produto estudado.

A solução 1 (Quadro 7) propiciou a redução de dois componentes (fios) e de um processo de soldagem deles ao adaptador. 0 adaptador anterior possuía modelos distintos para cada modelo de horímetro. Essa proposta permitiu que apenas um dos modelos existentes continuasse a ser utilizado, diminuindo a estrutura de componentes do produto e melhorando o planejamento da produção. A passagem de energia entre a placa de $\mathrm{Cl}$ do horímetro e o terminal passou a ser feito por duas molas, montadas internamente em alojamentos próprios na caneca. Esse processo de montagem é bem mais rápido do que o anterior, além do custo das molas ser inferior ao custo dos fios (incluindo corte e preparação) e também do tempo de inserção ser inferior àquele necessário para soldagem dos fios no adaptador.

A solução 2 propiciou, principalmente, facilidade de montagem dos dois conjuntos. 0 processo de solda foi eliminado, aproveitando-se do conceito de montagem por encaixe do produto de referência. Entretanto, o conceito utilizado na proposta de solução foi diferente do conceito encontrado no produto de referência. $\mathrm{Na}$ proposta, evitaram-se mudanças profundas nos componentes para evitar a necessidade de projeto de novos moldes para injeção dos componentes. Com o conceito utilizado, bastam apenas alguns pequenos ajustes nos moldes existentes para a produção dos novos componentes. Essa solução proposta, além de reduzir o tempo de montagem desses dois conjuntos, permite que sejam desmontados a qualquer momento durante o processo de montagem, no caso de não conformidade em qualquer dos outros componentes.

A solução 3 contribuiu na redução de custo de componente, uma vez que o mostrador não necessita mais ser produzido em alumínio, sendo que o novo componente pode ser produzido em aço zincado, assim como era o suporte do vidro. Além disso, a utilização de um adesivo para o mostrador elimina o processo de silk-screen e todos os subprocessos envolvidos (limpeza, pintura e silk), reduzindo o custo e o tempo de montagem. 0 novo componente diminui o número 
Quadro 7. Soluções propostas para reprojeto do horímetro.

\begin{tabular}{|c|c|c|}
\hline Componente & llustração & Solução proposta \\
\hline Solução 1: adaptador & & $\begin{array}{l}\text { Eliminação dos fios } \\
\text { Eliminação do processo de soldagem dos fios } \\
\text { Contato pelos terminais. }\end{array}$ \\
\hline $\begin{array}{l}\text { Solução 2: suporte principal e } \\
\text { suporte da bobina }\end{array}$ & & $\begin{array}{l}\text { Eliminação da solda para junção dos dois suportes } \\
\text { Facilidade da montagem por encaixe. }\end{array}$ \\
\hline $\begin{array}{l}\text { Solução 3: chapa de suporte do } \\
\text { vidro e mostrador }\end{array}$ & & $\begin{array}{l}\text { Um único componente ao invés de dois } \\
\text { Mostrador em aço ao invés de alumínio } \\
\text { Marcações com adesivo ao invés de silkscreen } \\
\text { Aletas para passagem de iluminação (deixando de ser feita por } \\
\text { lâmpadas e passando a ser feita por leds) } \\
\text { Pontos de fixação de referência para facilitar a montagem. }\end{array}$ \\
\hline Solução 4: caneca & & $\begin{array}{l}\text { Possibilidade de um único tipo de caneca, de uma única } \\
\text { dimensão, para atender a todos os clientes e para os produtos } \\
\text { com e sem iluminação. }\end{array}$ \\
\hline
\end{tabular}

de componentes, pois juntou dois componentes em um único (simplificação da estrutura do produto).

Por fim, a solução 4 foi um resultado natural das soluções anteriores, uma vez que foi possível propor uma única caneca como invólucro e proteção dos componentes do horimetro. Essa solução simplifica a estrutura do produto, favorecendo a padronização de componentes e reduz a possibilidade de falha na montagem por uso da caneca indevida para certo modelo de produto.

As melhorias propostas anteriormente representam uma redução significativa em diversos aspectos do produto e de seu processo de montagem/fabricação. Além disso, em caso de falhas nos testes, o horímetro poderá ser desmontado e trocadas somente as partes que não estiverem conforme, o que não é possível no horímetro atual.

Além da melhoria no tempo de montagem, as soluções propostas permitem uma redução significativa no custo-meta do horímetro, permitindo aumento de sua competitividade no mercado.

A Tabela 3 apresenta um resumo geral final dos benefícios obtidos com o produto reprojetado, não somente para as quatro propostas apresentadas no Quadro 7.

Em relação à redução do custo-meta do produto, a análise do grupo de pesquisa estimou uma redução da ordem de 33\%, propiciando oportunidades para a empresa competir com seus concorrentes diretos por participação no mercado.

Nas análises anteriores dos resultados finais desse reprojeto, não foram consideradas as melhorias realizadas na parte eletrônica do produto por ela não estar contemplada no escopo do presente trabalho.

0 procedimento empregado para a realização da integração da ER com o DFMA seguiu os passos propostos por Souza (2007), contribuindo para sua validação e generalização incremental, como ocorre com a pesquisa qualitativa, especialmente 
Tabela 3. Resumo dos resultados finais obtidos no reprojeto do horímetro.

\begin{tabular}{lccc}
\hline \multicolumn{1}{c}{ Conjunto } & $\begin{array}{c}\text { Redução } \\
\text { de } \\
\text { componentes } \\
(\%)\end{array}$ & $\begin{array}{c}\text { Redução } \\
\text { de } \\
\text { processos } \\
(\%)\end{array}$ & $\begin{array}{c}\text { Redução de } \\
\text { tempo de } \\
\text { montagem } \\
\text { e fabricação } \\
(\%)\end{array}$ \\
\hline Adaptador & 29 & 23 & 81 \\
Suporte completo & 29 & 36 & 44 \\
Embutimento - diversos & 17 & 0 & 30 \\
Embutimento - caixa & 17 & 24 & 62 \\
Embutimento - soquete & 100 & 100 & 100 \\
Embutimento - acessórios & 0 & 8 & 13 \\
Total & 11 & 15 & 47 \\
\hline
\end{tabular}

a pesquisa-ação. Os princípios da ER e do DFMA propostos por Ingle (1994), Stephenson e Wallace (1995), Matousek (1957) e Dufour (1996) mostraram-se eficazes para aplicação no presente trabalho.

\section{Conclusão}

Este trabalho apresentou uma pesquisa realizada com o apoio da Fapemig no Programa Primeiros Projetos e do Programa Pesquisador Mineiro.

Considera-se que os objetivos deste trabalho foram atingidos, uma vez que, a partir dos conceitos estudados na fundamentação teórica, foi possível analisar um modelo para o reprojeto de um dispositivo eletromecânico que proporcionou redução do número de componentes, do número de processos de montagem, do tempo de montagem/fabricação e do custo-meta do produto reprojetado.

As recomendações foram feitas com o cuidado de não alterar significativamente a infraestrutura atual da empresa em termos de máquinas e ferramentais, facilitando a incorporação das soluções apresentadas e não necessitando de nenhum grande investimento para a sua implantação.

A pesquisa também mostrou que a integração do projeto para manufatura e montagem (DFMA) com a prototipagem rápida, em uma abordagem de engenharia reversa, tal como proposta por Souza (2007), é uma estratégia adequada para a melhoria (reprojeto) de produtos de empresas de pequeno porte. A engenharia reversa permite o estudo de outras tecnologias ou soluções para manufatura ou montagem empregadas por concorrentes. Já o DFMA permite que essas tecnologias ou conceitos possam ser adaptados para a simplificação, padronização de componentes, redução de operações secundárias, entre outras.

0 modelo proposto por Souza (2007) mostrou-se consistente para a realização de um reprojeto de um produto eletromecânico. Espera-se que este presente trabalho possa contribuir para a validação incremental desse modelo. Recomenda-se que outros pesquisadores utilizem este mesmo modelo em outras pesquisas similares, visando uma possível generalização no futuro.

\section{Referências}

ANDREASEN, M. M. Design for production - overview of methodology. In: INTERNATIONAL CONFERENCE ON ENGINEERING DESIGN - ICED'91, 1991, Zurich. Proceedings... 1991. p. 516-521.

BACK, N. et al. Projeto integrado de produtos: planejamento, concepção e modelagem. Porto Alegre: Manole, 2008.

BACK, N. Metodologia de projeto de produtos industriais. Rio de Janeiro: Guanabara Dois, 1983.

CHEN, L. C. Reverse engineering in the design of turbine blades - a case study in applying the MAMDP. Robotics and Computer Integrated Manufacturing, v. 16, n. 2-3, p. 161-167, 2000. http://dx.doi.org/10.1016/S07365845(99)00044-7

CHOI, S. H.; CHAN, A. M. M. A virtual prototyping system for rapid product development. Computer-Aided Design, v. 36, p. 401-412, 2004. http://dx.doi.org/10.1016/ S0010-4485(03)00110-6

COOPER, R. G. Stage-gate systems: a new tool for managing new products. Business Horizons, 1990.

COOPER, R. G.; EDGETT, S. J. Product development for de service sector. Lessons from market leaders. New York: Basic Books, 1999.

COUGHLAN, P.; COGHLAN, D. Action research for operations management. International Journal of Operations \& Production Management, v. 22, n. 2, p. 220-240, 2002. http://dx.doi.org/10.1108/01443570210417515

DIAS, A. B. Engenharia reversa: uma porta ainda aberta. Produto Et Produção, v. 2, n. 1, p. 1-7, 1998.

DUFOUR, C. A. Estudo do processo e das ferramentas de reprojeto de produtos industriais, como vantagem competitiva e estratégia de melhoria constante. 1996, 122 f. Dissertação (Mestrado)-Universidade Federal de Santa Catarina, Florianópolis, 1996.

FERREIRA, H. S. R.; TOLEDO, J. C. Metodologias e ferramentas de suporte à gestão do processo de desenvolvimento de produto (PDP) na indústria brasileira de autopeças. In: CONGRESSO BRASILEIRO DE GESTÃO DE DESENVOLVIMENTO DE PRODUTO, 3., 2001, Florianópolis. Anais... 2001.

FREIXO, O. M. Incorporação da gestão dos custos do ciclo de vida ao processo de desenvolvimento do produto da Embraer. Tese (Doutorado em Engenharia de Produção)Universidade Federal de São Carlos, São Carlos, 2004.

GRIFFIN, A.; PAGE, A. L. PDMA success measurement project: recommended measures for product development success and failure. Journal of Innovative Management, v. 13, p. 478-496, 1996. http://dx.doi.org/10.1016/S07376782(96)00052-5

HARMSEN, H.; GRUNERT, K. G.; BOVE, K. Company competencies as a network: the role of product development. Journal of Product Innovation Management, v. 17, p. 197-207, 2000. http://dx.doi. org/10.1016/S0737-6782(00)00039-4 
HOBDAY, M.; RUSH, H.; BESSANT, J. Approaching the innovation frontier in Korea: the transition phase to leadership. Research Policy, v. 33, p. 1433-1457, 2004. http://dx.doi.org/10.1016/j.respol.2004.05.005

HUANG, G. Q.; MAK, K. L. Design for manufacture and assembly on the Internet. Computers in Industry, v. 38, p. 17-30, 1998. http://dx.doi.org/10.1016/S01663615(98)00105-5

INGLE, K. A. Reverse engineering. New York: McGrawHill, 1994. 240 p.

KAMINSKI, P. C. Desenvolvendo produtos com planejamento, criatividade e qualidade. Rio de Janeiro: LTC, 2000.

KIM, L.; NELSON, R. Tecnologia, aprendizado e inovação: as experiências das economias de industrialização recente. Campinas: Unicamp, 2005.

KOCHAN, A. Rapid prototyping gains speed, volume and precision. Assembly Automation, v. 20, n. 4, p. 295-299, 2000. http://dx.doi. org/10.1108/01445150010378425

MATOUSEK, R. Konstruktionslehre des allgemeinen Maschinenbaues. Berlin: Springer, 1957.

MELLO, C. H. P.et al . Projeto conceitual de componentes de um forno industrial por meio da integração entre a engenharia reversa e o DFMA. Gestão e Produção, v. 17 , n. 3, p. 497-511, 2010. http://dx.doi.org/10.1590/ S0104-530X2010000300005

MIGUEL, P. A C. QFD no desenvolvimento de novos produtos: um estudo sobre a sua introdução em uma empresa adotando a pesquisa-ação como abordagem metodológica. Produção, v. 19, n. 1, p. 105-128, 2009. http://dx.doi.org/10.1590/S0103-65132009000100008

MURY, L. G. M. Uma metodologia para adaptação e melhoria de produtos a partir da engenharia reversa. 2000. 89 f. Dissertação (Mestrado em Engenharia de Produção)Universidade Federal do Rio Grande do Sul, Escola de Engenharia, Porto Alegre, 2000.

MYRUP, A. Design for production - Overview of methodology. In: INTERNATIONAL CONFERENCE ON ENGINEERING DESIGN - ICED 91, 1991, August. Anais...1991.
OGLIARI, A. Sistematização da concepção de produtos auxiliada por computador com aplicações no domínio de componentes de plástico injetado. Tese (Doutorado)-Universidade Federal de Santa Catarina, Florianópolis, 1999.

OTTO, K. N.; WOOD, K. L. Product evolution: a reverse engineering and redesign methodology. Research in Engineering Design, v. 10, p. 226-243, 1998. http:// dx.doi.org/10.1007/s001639870003

PAHL, G.et al. Engineering design: a systematic approach. Springer, 2005.

ROSENTHAL, S. R. Effective product design and development - How to cut lead time and increase customer satisfaction. New York: Irwin Professional Publishing, 1992.

ROZENFELD, H.et al. Gestão de desenvolvimento de Produtos - uma referência para a melhoria do processo. São Paulo: Saraiva, 2006.

SILVA, C. E. S. Método para avaliação do desempenho do processo de desenvolvimento de produtos. Tese (Doutorado em Engenharia de Produção)-Universidade Federal de Santa Catarina, Florianópolis, 2001.

SOUZA, J. F. Aplicação de projeto para manufatura e montagem em uma abordagem de engenharia reversa: estudo de caso. 2007. 135 f. Dissertação (Mestrado em Engenharia de Produção)-Universidade Federal de Itajubá, ltajubá, 2007.

STEPHENSON, J.; WALLACE, K. Design for reability in mechanical systems. In: INTERNATIONAL CONFERENCE ON ENGINEERING DESIGN - ICED 95. 1995, Praha. Anais...1995.

THIOLLENT, M. Metodologia da pesquisa-ação. 15. ed. São Paulo: Cortez Editora, 2007. 132 p.

VINCENT, G. Managing new product development. New York: Van Nostrand Reinold, 1989.

WHEELWRIGHT, S. C.; CLARK, K. B. Revolutionizing product development - Quantum leaps in speed, efficiency, and quality. New York: Free Press, 1992.

ZHU, J.; LIANG, X.; XU, Q. The cause of secondary innovation dilemma in chinese enterprises and solutions. In: INTERNATIONAL ENGINEERING CONFERENCE IEEE, 2005. Proceedings... 2005. v. 1, p. 297-301.

\title{
Redesign of an electromechanical device in a reverse engineering approach integrated to design for manufacturing and assembly and rapid prototyping
}

\begin{abstract}
The purpose of this work was the study of the integration between design for manufacturing and assembly with rapid prototyping, in a reverse engineering approach, such as some tools to support product redesign. Based on a literature review of these concepts, this paper aimed at examining the application of a model that integrates the design for manufacture and assembly with rapid prototyping in reverse engineering approach in the redesign of an electromechanical device. The objective was to analyze the results of the application of this model in order to reduce the time of manufacture/assembly and the cost of assembly in the redesign of the product, which has been marketed for more than 30 years. The research method employed was action-research, because the researcher wanted to address a problem identified within the object of study, in partnership with the company's research team. The results were recommendations for the redesigning of the product, which showed a reduction in the assembly time, in the number of processes and components, and also in the targeted cost of the product - providing the opportunity to improve the competitiveness of the company in the market.
\end{abstract}

\section{Keywords}

Reverse engineering. DFMA. Rapid prototyping. Redesign. 